\title{
Clostridium sticklandii, a specialist in amino acid degradation:revisiting its metabolism through its genome sequence
}

\author{
Nuria Fonknechten ${ }^{1,2,3}$, Sébastien Chaussonnerie ${ }^{1,2,3}$, Sabine Tricot ${ }^{1,2,3}$, Aurélie Lajus ${ }^{1,2,3}$, Jan R Andreesen ${ }^{5}$, \\ Nadia Perchat ${ }^{1,2,3}$, Eric Pelletier ${ }^{1,2,3}$, Michel Gouyvenoux ${ }^{1,2,3}$, Valérie Barbe ${ }^{1,2,3}$, Marcel Salanoubat ${ }^{1,2,3}$, \\ Denis Le Paslier ${ }^{1,2,3}$, Jean Weissenbach ${ }^{1,2,3}$, Georges N Cohen ${ }^{4}$, Annett Kreimeyer ${ }^{1,2,3^{*}}$
}

\begin{abstract}
Background: Clostridium sticklandii belongs to a cluster of non-pathogenic proteolytic clostridia which utilize amino acids as carbon and energy sources. Isolated by T.C. Stadtman in 1954, it has been generally regarded as a "gold mine" for novel biochemical reactions and is used as a model organism for studying metabolic aspects such as the Stickland reaction, coenzyme-B12- and selenium-dependent reactions of amino acids. With the goal of revisiting its carbon, nitrogen, and energy metabolism, and comparing studies with other clostridia, its genome has been sequenced and analyzed.

Results: C. sticklandii is one of the best biochemically studied proteolytic clostridial species. Useful additional information has been obtained from the sequencing and annotation of its genome, which is presented in this paper. Besides, experimental procedures reveal that C. sticklandii degrades amino acids in a preferential and sequential way. The organism prefers threonine, arginine, serine, cysteine, proline, and glycine, whereas glutamate, aspartate and alanine are excreted. Energy conservation is primarily obtained by substrate-level phosphorylation in fermentative pathways. The reactions catalyzed by different ferredoxin oxidoreductases and the exergonic NADH-dependent reduction of crotonyl-CoA point to a possible chemiosmotic energy conservation via the Rnf complex. C. sticklandii possesses both the F-type and V-type ATPases. The discovery of an as yet unrecognized selenoprotein in the D-proline reductase operon suggests a more detailed mechanism for $\mathrm{NADH}$-dependent D-proline reduction. A rather unusual metabolic feature is the presence of genes for all the enzymes involved in two different $\mathrm{CO}_{2}$-fixation pathways: C. sticklandii harbours both the glycine synthase/glycine reductase and the Wood-Ljungdahl pathways. This unusual pathway combination has retrospectively been observed in only four other sequenced microorganisms.

Conclusions: Analysis of the C. sticklandii genome and additional experimental procedures have improved our understanding of anaerobic amino acid degradation. Several specific metabolic features have been detected, some of which are very unusual for anaerobic fermenting bacteria. Comparative genomics has provided the opportunity to study the lifestyle of pathogenic and non-pathogenic clostridial species as well as to elucidate the difference in metabolic features between clostridia and other anaerobes.
\end{abstract}

\section{Background}

Amino acids are used as important carbon and energy sources for some microorganisms. This strategy represents an advantage in protein-rich environments, but it is used even when crucial metabolic processes (e.g. protein

\footnotetext{
* Correspondence: akreimey@genoscope.cns.fr

'CEA, DSV, Institut de Génomique, Genoscope, 2 rue Gaston Crémieux, F-

91057 Evry, France

Full list of author information is available at the end of the article
}

biosynthesis), may be impaired. A variety of anaerobic bacteria have developed specific pathways to degrade amino acids by fermentation processes. Anaerobic amino acid utilization was extensively studied in the seventies, especially in Clostridia [1,2]. The genus Clostridium consists of a large group of Gram-positive, anaerobic bacteria which belong to the Firmicute phylum [3]. This genus employs pathways and enzymes with unique activities mostly involved in amino acid degradation, such as
C Biomed Central

(c) 2010 Fonknechten et al; licensee BioMed Central Ltd. This is an Open Access article distributed under the terms of the Creative Commons Attribution License (http://creativecommons.org/licenses/by/2.0), which permits unrestricted use, distribution, and reproduction in any medium, provided the original work is properly cited. 
B12-dependent aminomutases, selenium containing oxidoreductases and extremely oxygen-sensitive 2-hydroxyacyl-CoA dehydratases [4-6]. A striking metabolic feature of Clostridia is the fermentation of amino acids via the Stickland reaction, described in 1934 by L. H. Stickland [7]. It is characterized by the oxidation of one amino acid coupled to the reduction of another. In this process energy is mainly conserved by ATP formation via substrate-level phosphorylation (SLP). However, little is known about other systems by which the Stickland reaction contributes to energy and growth, despite many studies of this aspect of clostridial metabolism [6,8-11]. In this context, one of the best biochemically studied clostridial species is Clostridium sticklandii. After its isolation from San Francisco Bay black mud in 1954 [12,13], it was described to be a specialist in amino acid degradation $[14,15]$. More specifically, C. sticklandii can utilize threonine, arginine, lysine and serine as reductants in the Stickland reaction, whereas glycine and proline are used as oxidants $[1,2,16]$. It has been described that ornithine/ proline or ornithine/lysine can be used as amino acid pairs in the Stickland reaction. Aromatic and branchedchain amino acids also seem to be degraded, but the pathways involved are still unknown $[17,18]$. Two amino acids, glutamate and alanine, are not utilized. Stadtman has stated that formate, when added to the amino acid fermentations, increased growth yield [12,16]. Besides the degradation of amino acids, it has also been reported that C. sticklandii catabolises purines $[19,20]$ and slightly ferments carbohydrates such as glucose, maltose and galactose [21]. However, these compounds are not or only minor substrates for energy and growth.

To get a general view of amino acid catabolising microorganisms, we performed a preliminary bioinformatic analysis (Blast searches) of complete sequenced bacterial genomes in which specific enzymes involved in amino acid degradation pathways are present. The results showed that Clostridium spp. possess most of these pathways (data not shown). Since the biochemistry of amino acid fermentation has been principally studied in C. sticklandii, this bacterium is an appropriate candidate for learning more about the fascinating process of anaerobic amino acid degradation. We have sequenced and analyzed its genome. Together with supplemental experimental procedures these studies have confirmed and extended our knowledge of amino acid degradation. They have also revealed previously unknown metabolic capacities of $C$. sticklandii that contribute to an improved understanding of its energyconserving system, particularly the processes of chemiosmotic ATP generation. Moreover, a hitherto unrecognized selenoprotein and an unusual combination of two distinct $\mathrm{CO}_{2}$-fixation pathways have been discovered. Finally, this work has provided the opportunity for a comparative study of other clostridial genomes, notably the most closely related pathogenic strain, Clostridium difficile $[6,21,22]$. Together, these results help to elucidate the biochemistry, energetics and growth of clostridia and other anaerobic amino acid degrading bacteria.

\section{Results and discussion}

\section{Global genomic and metabolic features}

The major features of the C. sticklandii genome are listed in Table 1 (comparison with other clostridial species - Additional file 1). Its genome consists of a single circular chromosome of 2,715,461 bp, which carries 2573 coding sequences (CDS). The number of CDS in such a small genome and the low level of repeated regions (2.1\%) explain the high protein coding density of $89.2 \%$. Of the 2573 CDS, $33 \%$ were annotated as conserved hypothetical or hypothetical proteins. Two members of the genus Alkaliphilus (A. metalliredigens QIMF and $A$. oremlandii OhILas) and Clostridium difficile 630 share the highest number of orthologous genes with C. sticklandii (Additional file 2).

Annotation of the C. sticklandii genome reveals that most biosynthetic pathways considered as essential for viability seem to be present (e.g., biosynthesis of amino acids, purines, pyrimidines and cofactors). However, it should be noted that genes involved in synthesis of biotin and pantothenate are absent, confirming the absolute requirement of these two vitamins for growth observed by Golovchenko et al. [23]. These authors also report a requirement for cyanocobalamin and thiamin. The absence of some genes involved in the biosynthesis of these cofactors is in agreement with these results. Pyridoxal phosphate synthesis proceeds through the proteins encoded by $p d x S$ and $p d x T$ [24] and not by the pathway existing in E. coli and many other bacteria. C. sticklandii is unable to assimilate sulphate, since genes involved in this pathway are absent $(c y s N$, cys $D, c y s C$, and $c y s H$ ). This inability is also found in some other clostridia (Additional file 1). In C. sticklandii, the sulphur requirement for cysteine biosynthesis can be met by sulphide (data not shown) since genes coding for serine $\mathrm{O}$-acetyltransferase (cysE) and O-acetylsulfhydrylase (cysK)

Table 1 Genomic features of the C. sticklandii DSM 519 genome

\begin{tabular}{lc}
\hline Features & \\
\hline Complete genome size, Mb & 2.7 \\
G+C content (\%) & 33.3 \\
Repeat regions (\%) & 2.1 \\
Number of CDS & 2573 \\
Protein coding density (\%) & 89.2 \\
Average CDS length (bp) & 945 \\
Number of tRNAs & 59 \\
Number of rRNA clusters & 6 \\
\hline
\end{tabular}


are present. The only genes of the upper portion of the tricarboxylic acid cycle found with certainty in this bacterium are the adjacent genes encoding $\mathrm{Si}$-citrate synthase and isocitrate dehydrogenase. Since glutamate is excreted (see section below), there is no need for glutamate formation via the tricarboxylic acid cycle. Glutamate could be formed by histidine degradation since all the genes coding for the enzymes of histidine biosynthesis and degradation are present. Additionally, 2-ketoglutarate could be formed by reductive carboxylation of succinyl-CoA using reduced ferredoxin by one of the many 2-ketoacid ferredoxin oxidoreductases. All genes involved in the glycolysis pathway were detected. However, we found that C. sticklandii is not able to grow on glucose (data not shown) although a slight fermentation of glucose has been described [21]. This is explained by a defect in some transport components [25]. Indeed, genome analysis shows the absence of genes coding for protein II of the PTS system. The genes of the non-oxidative pentose phosphate pathway are present, accounting for the synthesis of hexose and pentose phosphates respectively. A cluster of genes encoding a ribose transporter and a ribokinase could lead to ribose 5 -phosphate, which can be completely converted into glycolytic intermediates.

As a proteolytic species, C. sticklandii contains about 12 proteases and 38 peptidases and is highly dependent on the utilization of amino acids (see section below). Annotation of its genome has confirmed the presence of several biochemically described amino acid degradation pathways (Additional file 3). Similar amino acid degradation pathways are found in the metal-reducing strain Alkaliphilus metalliredigens QIMF, in the arsenic-resistant strain Alkaliphilus oremlandii OhILas [26,27] as well as in the pathogenic strains of $C$. difficile and C. botulinum (Additional file 4). It should be noticed that arginine deiminase is missing in the genome of $C$. difficile, although this species contains all the genes involved in the subsequent steps in the arginine breakdown pathway. The genes coding for several transporters for amino acids were found in C. sticklandii (e.g., ABC transporters for oligopeptides, methionine, and branched-chain amino acids, a serine/ threonine symporter as well as an arginine/ornithine antiporter). The degradation of the purines (adenine, xanthine and uric acid) by C. sticklandii has been reported $[19,20]$. However, xanthine dehydrogenase, the key enzyme of purine degradation, was not found in this genome. We cannot exclude that purine compounds are converted by a totally different 2-ketopurine dehydrogenase. This protein has been purified from several species of peptostreptococci [28], but the gene is not known.

\section{Preferential and sequential amino acid utilization}

Important features of amino acid metabolism by C. sticklandii are depicted in Figure 1A. It oxidizes ornithine, arginine, threonine, serine and reduces proline and glycine $[2,12,15]$. C. sticklandii can utilize glycine and ornithine both as an electron donor and an electron acceptor in the Stickland reaction. Ornithine can be reduced to 5-aminovaleric acid through the formation of D-proline as an intermediate, or it can be oxidized to acetate, D-alanine and ammonia $[15,29,30]$. Glycine can be directly oxidized by $\mathrm{NAD}^{+}$into methylene-THF, $\mathrm{CO}_{2}$ and ammonia via the glycine cleavage system (gcv-system) or reduced to acetyl phosphate by glycine reductase [31]. Electrons from the gcv-system can be directly utilized by glycine reductase as observed for Eubacterium acidaminophilum [32]

Since a large variety of amino acids can be utilized by C. sticklandii as nutrient and energy sources, it was interesting to determine which amino acids are preferentially metabolized by this bacterium. For this purpose C. sticklandii was cultivated in a defined medium with the amino acids at the same concentration (see Methods). Using Liquid Chromatography-Mass Spectrometry (LC-MS) analysis (Figure 1B and Additional file 5) we found that arginine, serine, threonine, cysteine, proline and glycine disappear rapidly from the medium in the exponential growth phase. In contrast, lysine, histidine, asparagine and valine disappear at the onset of the stationary growth phase. The concentrations of the aromatic amino acids remained stable: they were not metabolized under our growth conditions. Glutamate, aspartate and L- and D-alanine are excreted. These results show that $C$. sticklandii seems to prefer certain amino acids and degrades them in a specific order.

Subsequently, we tried to associate amino acid utilization with the analysis of genes coding for proteins involved in the degradation pathways. The rapid utilization of threonine can be explained by the presence of three pathways through which this amino acid is catabolized. Threonine dehydrogenase oxidizes threonine into 2-amino-3-ketobutyrate [33] which is split into glycine and acetyl-CoA. Threonine aldolase converts threonine into glycine and acetaldehyde, whereas threonine dehydratase transforms threonine into ammonia and 2-ketobutyrate. The latter compound is oxidized to propionate and $\mathrm{CO}_{2}$ or reductively aminated to 2 -aminobutyrate (Figure 1A), typically formed by $C$. sticklandii [1] and detected in extracellular medium by LC-MS (data not shown). Arginine is quickly metabolized through the arginine deiminase pathway into ornithine via citrulline. This is a generally favoured pathway by anaerobes, for they can conserve energy without any redox reaction by splitting the deaminated intermediate citrulline by ornithine carbamoyl-phosphate transferase and conserve ATP by carbamate kinase. As expected, C. sticklandii contains all the genes involved in the arginine deiminase pathway as well as the ornithine oxidative and reductive 


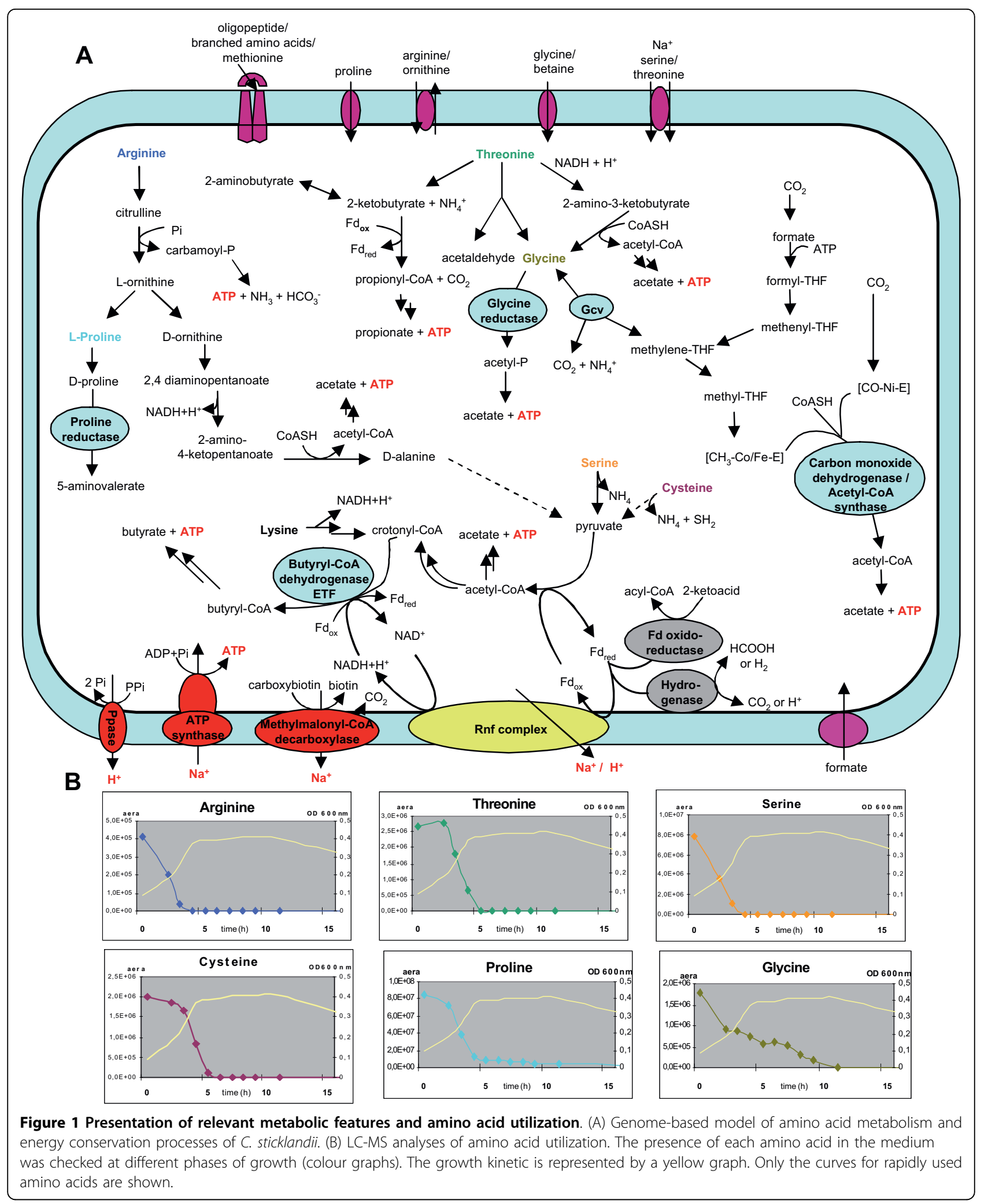


pathways [29,30]. Like C. botulinum and C. sporogenes [34], C. sticklandii possesses an ornithine cyclodeaminase that directly produces proline from ornithine. Dehydration of serine leads to pyruvate which is decarboxylated into acetyl-CoA by pyruvate ferredoxin oxidoreductase. The rapid utilization of cysteine may be correlated with its degradation to pyruvate, ammonia and hydrogen sulphide by a putative L-cysteine sulphide lyase. In the degradation of lysine into acetate, butyrate, and ammonia [35-37], the highly exergonic reduction of crotonyl-CoA to butyryl-CoA contributes to energy conservation [38]. However, lysine is not used in the exponential growth phase (Additional file 5). One could hypothesize that during this growth phase crotonyl-CoA is formed from the condensation of two acetyl-CoAs via butyrate fermentation, since all the genes involved in this pathway are present in C. sticklandii. Acetyl-CoA could be generated from serine, arginine and cysteine during the exponential growth phase. Thus, after depletion of these amino acids (at the beginning of the stationary growth phase) crotonyl-CoA is produced from lysine fermentation. It has been reported that C. sticklandii degrades aromatic and branched chain amino acids by oxidative means $[14,17,18]$. However, under our experimental conditions, aromatic amino acids do not appear to be metabolized (Additional file 5) whereas valine, leucine, and (or) isoleucine (the two latter compounds are not distinguishable by mass spectrometry) disappear from the medium. They are probably transaminated to the corresponding 2-ketoacids, which are further degraded by the branched-chain fatty acid ferredoxin oxidoreductase. Alanine, which was not added to the medium, may be formed by the breakdown of ornithine. This amino acid is excreted in the exponential phase, but disappears slowly in the stationary growth phase. Stadtman has shown that the Stickland reaction amino acid pair, alanine and glycine, is not operative in C. sticklandii [16]. A gene coding for alanine dehydrogenase is actually absent in C. sticklandii. Glutamate might be formed by degradation of histidine but in contrast to other clostridial species, especially $C$. tetanomorphum and C. cochlearium [39], glutamate is not used by C. sticklandii in accordance with previous results $[1,2]$. Genes encoding methylaspartate mutase or 2-hydroxyglutaryl-CoA dehydratase, the key enzymes of the two major glutamate fermentation pathways [40], have not been detected in the genome.

Based on the results of amino acid utilization, we tried to grow C. sticklandii in three different media. The following amino acid combinations were utilized: the first contained amino acids utilized in the exponential growth phase, the second contained those utilized in the exponential and stationary growth phase and the third was equivalent to the second complemented with amino acids that apparently were not metabolized (Additional file 6). Surprisingly, no growth was observed in the medium with the second amino acid combination. Similar experiments have been performed by Golovchenko et al. using C. sticklandii strain CSG [23]. Thus, it appears that the combination of amino acid influences the growth of C. sticklandii. There are probably regulatory properties implying that the presence or absence of some amino acids could repress the utilization or the biosynthesis of other amino acids. In particular, it has been shown that in some clostridial species that perform the Stickland reaction, the type of amino acid pair is quite important for growth [41].

\section{Discovery of PrdC as a selenocysteine-containing protein}

Selenoproteins are characterized by the replacement of a cysteine residue by a selenocysteine ( $\mathrm{Sec}$ ) encoded by the UGA stop-codon. A complex machinery is required for translational insertion of Sec in response to the UGA codon. In fact, the first selenoprotein, glycine reductase A (GrdA), was discovered in C. sticklandii $[42,43]$. Analysis of the C. sticklandii genome revealed that all known components of the Sec insertion machinery (Additional file 3) and the Sec tRNA are present. Using the gene fusion-fission functionality of MaGe [44], we identified eight selenoproteins in C. sticklandii (Table 2). Interestingly, one of these proteins, PrdC, had never been described as a selenoprotein before, although the proline reductase genes have been studied in detail in both $C$. difficile and C. sticklandii $[22,45,46]$. PrdC is located in the D-proline reductase operon and is annotated as an electron transfer protein [45]. This role has also been suggested by Eversmann [47]. Evidence for the exact biochemical role of $\mathrm{PrdC}$ is now provided by the discovery of a C-terminal redox center -CxxU-. The amino acid sequence of PrdC exhibits homology to $\mathrm{RnfC}$, the protein involved in the NADH-dependent Rnf electron transfer complex. Recently, Kim et al. have

Table 2 Selenocysteine containing proteins in $C$. sticklandii

\begin{tabular}{lcc}
\hline Proteins & CDS number & $\begin{array}{c}\text { Sec position } \\
\text { (protein length) }\end{array}$ \\
\hline
\end{tabular}

Known selenoproteins:

Formate dehydrogenase (FdhA)

Betaine reductase $(\mathrm{GrdH})$

Glycine reductase (GrdA)

Glycine reductase (GrdB)

Proline reductase (PrdB)

Peroxiredoxin

Putative glutathione peroxidase

CLOST_0829

$134(699)$

CLOST_1057

$352(440)$

CLOST_1112

44 (158)

CLOST_1113

$350(436)$

CLOST_2232

$152(242)$

CLOST_2406

84 (240)

New selenoprotein:

CLOST_2446

$35(161)$

Electron transfer protein ( $\mathrm{PrdC}$ 
identified RnfC as a selenoprotein in Alkaliphilus oremlandii OhILAs (accession no. YP_001511593). However, the genomic context and the alignment of this sequence with the PrdC of several clostridial species (Figure 2) show that this selenoprotein $(\mathrm{RnfC})$ is actually PrdC. This alignment also shows that the selenocysteine-residue is present in all PrdC proteins in the terminal -CxxU-motif with two exceptions: there is a deviation of the selenocysteine position in PrdC of C. botulinum, and $\operatorname{PrdC}$ of $C$. difficile does not contain a selenocysteine but a cysteine residue, thus emphasizing that the presence of selenocysteine in $\operatorname{PrdC}$ is not a necessity, but is probably catalytically advantageous as has been shown e.g. for methionine sulphoxide reductases [48].

\section{New insights into the D-proline reductase mechanism}

D-Proline acts as the electron acceptor in the Stickland reaction. The $\mathrm{D}$-proline reductase has been biochemically studied only in C. sticklandii [46,49] and in C. difficile [22]. It catalyzes the reductive ring cleavage of D-proline into 5-aminovalerate, which is subsequently excreted. In C. sporogenes, the reduction of D-proline was described to be coupled to transmembrane proton ejection. However, it has not been demonstrated whether this proton gradient is coupled to ATP synthesis [50]. This is possibly due to a very low proton ejection. The D-proline reductase activity of C. sticklandii was detected in the cytoplasmic fraction [46]. In contrast to ATP formation by the glycine reductase reaction [8], no obvious energy conservation step was identified in the D-proline reductase reaction $[45,46,51]$. Surprisingly, despite this energetic disadvantage, $C$. sticklandii preferentially uses proline when glycine and proline are both present in the synthetic medium $[31,52]$. It seems likely that D-proline reductase is used as an electron sink and plays a role in the redox-balance of the cell
[51]. NADH has been suggested as the natural electron donor [51,53]. Genes involved in D-proline reduction are clustered together in an operon and the organization of the genes is conserved in all the organisms studied (Additional file 7). PrdF encodes the proline racemase necessary for D-proline generation in order to discriminate it from the L-proline used for protein synthesis. PrdA undergoes a post-translational modification forming a catalytically active pyruvoyl group $[47,54]$ that binds the D-proline $[45,46]$ (Figure 3). The dissociated, nucleophilic selenocysteine moiety in PrdB could then attack the $\alpha$-C-atom of proline. This may lead to a reductive cleavage of the $\mathrm{C}$ - $\mathrm{N}$-bond of the pyrrolidine ring and the formation of a selenoether. The selenoether would then be cleaved by a cysteine moiety of PrdB resulting in a mixed selenide-sulphide group. PrdC contains NADH- and FMN-binding sites as well as an ironsulphur cluster as suggested before $[46,51,53]$ and has been hypothesized to function as an electron transport protein $[45,47]$. With the detection of the potential redox-active center including a selenocysteine in $\operatorname{PrdC}$, we now propose the following extension of the reaction: PrdC will use NADH to reduce its special terminal sulphide-selenide group into the thiol-selenol form (Figure 3). The latter seems to be optimal to reduce the oxidized selenide-sulphide bond of PrdB by an exchange reaction. A similar type of dithiol-disulphide exchange reaction (partly with an involvement of selenocysteine) occurs during glycine reduction by NADPH via thioredoxin reductase, thioredoxin, GrdA and GrdB, where selenocysteine replaces one cysteine in GrdA and GrdB [8,55]. PrdD and PrdE correspond quite significantly in their sequences to the middle and C-terminal part of proprotein PrdA. Both genes are present in an operonlike structure in all genomes of organisms containing a D-proline reductase (Additional file 7). However, both

\section{C. sticklandii \\ A. metalliredigens \\ A. oremlandii \\ C. difficile \\ C. botulinum
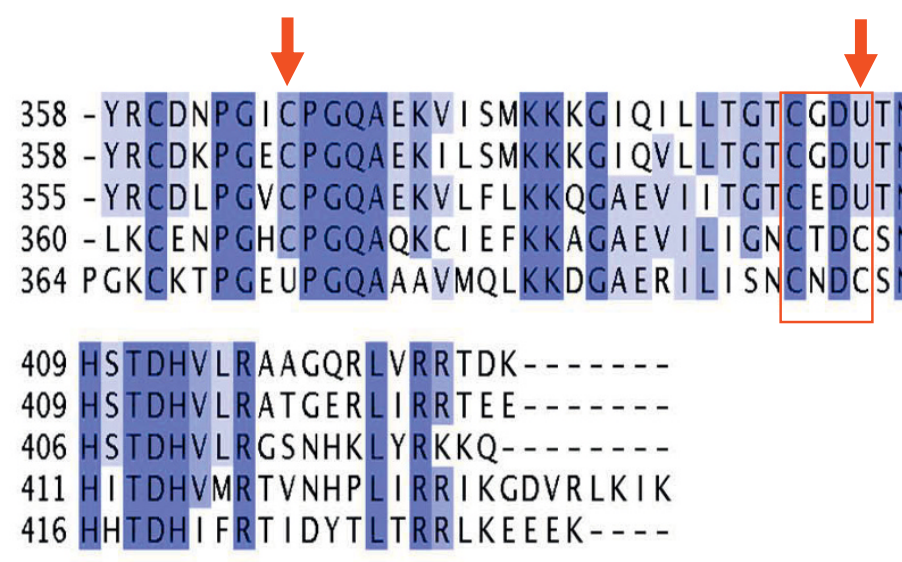 \\ 358 - YRCDNPCI CPGQAEKV I SMKKKCIQI LLTCTICDUTNTVMGVAPRLGVPTY 408 358 - YRCDKPCECPGQAEK I LSMKKKG I QVLLTCT CCDUTNTVMNVAPRLGVPTY 408 355 - YRCDLPCVCPGQAEKVLF LKKQGAEV I I TCT CEDUTNTVMMTAPRLCVPVY 405 360 - LKCENP CHCP GQAQKC I EFKKACAEV I L I GNCTDCSNTVMGSAPKLKLCVY 410 364 PCKCKTPCEUPCQAAAVMQLKKDGAERILISNCLNDSNTVMQIAPKAKIPVY 45

Figure 2 Multiple sequence alignments of the C-terminal sequence of PrdC of several Clostridiales. Conserved residues are highlighted. The red arrows show the position of the selenocysteine $(U)$. The redox centre is boxed in red. 


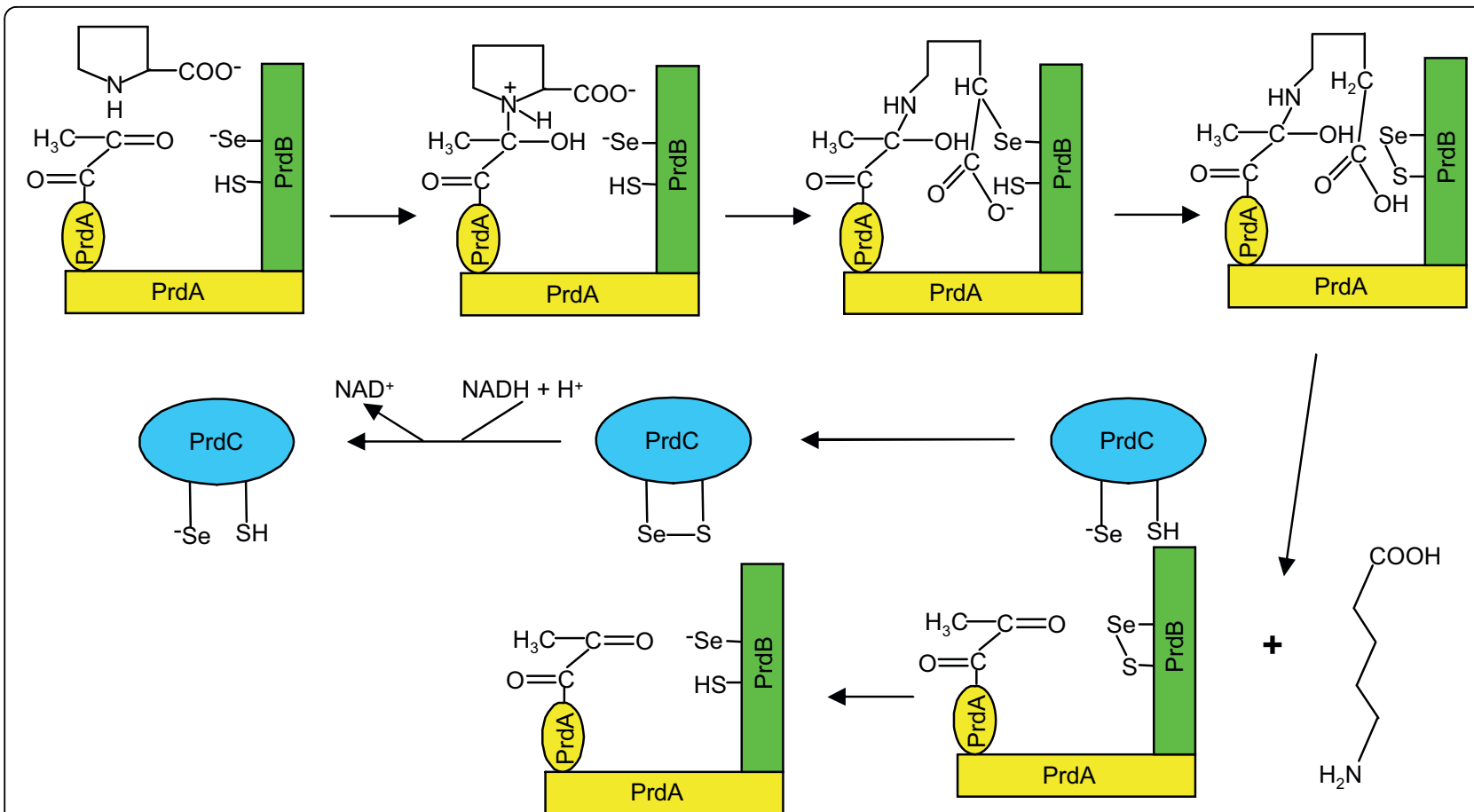

Figure 3 Proposed mechanism of D-proline reductase (adapted from Kabisch [45]). PrdA is shown after the hydrolytic processing of the proprotein to form a pyruvoyl group that binds D-proline [46].

genes are absent in the second set of D-proline reductase genes in C. botulinum, pointing to a more indirect, protein-stabilizing role as shown for the related two proteins derived from proprotein GrdE in glycine reductase $[8,55]$. Finally, 5 -aminovalerate is formed by hydrolysis to regenerate the pyruvoyl moiety [46]. PrdG is a potential membrane-bound protein and might be responsible for the excretion of 5-aminovalerate.

\section{Oxidative stress response}

Clostridia are described in textbooks as obligate anaerobes since oxygen is harmful or lethal to these bacteria. For example, pyruvate ferredoxin oxidoreductase, a central enzyme of their metabolism is quickly inactivated in the presence of oxygen. Apparently, this results from damage to its exposed iron-sulphur sites [56]. However, some obligate anaerobes can tolerate small amounts of oxygen thanks to the presence of some proteins involved in oxygen detoxification [10]. In C. acetobutylicum genes coding for some of these proteins are under control of the peroxide repressor PerR, whose deletion results in a prolonged aerotolerance [57]. Genome analysis of $C$. sticklandii has revealed the presence of a protein similar to PerR as well as several proteins involved in the oxidative stress response. Beside the classical $\mathrm{Mn}$-superoxide dismutase and superoxide reductase, $C$. sticklandii possesses genes encoding an alkyl hydroperoxide reductase, a regular rubrerythrin, two putative glutathione peroxidases, a seleno-peroxiredoxin as well as a thioredoxin-dependent peroxidase. The methionine sulfoxide reductases A and B involved in the repair of oxidative damaged methionine have also been detected. This well-supplied protection system could enhance the survival of $C$. sticklandii under microaerophilic conditions. In fact, we observed slight growth when C. sticklandii was cultivated in an aerobic atmosphere (data not shown).

\section{Energy conservation}

The energy conservation of $C$. sticklandii via amino acid fermentation and especially by the Stickland reaction, has been the subject of many investigations. In this context, glycine reductase seems to play a central role [8]. The reduction of glycine leads to ATP formation by SLP with acetate as the final product. SLP is also an important step in some fermentation processes (Figure 1A). Energy conservation via electron-transport phosphorylation (ETP) in C. sticklandii is performed by the Rnf complex. This complex, that was recently discovered in various bacteria and archaea, is a membrane-bound system showing strong similarity to the Rhodobacter capsulatus nitrogen fixation and some NADH:quinone oxidoreductase systems. It catalyzes the electron flow from reduced ferredoxin to $\mathrm{NAD}^{+}$. This process is coupled to ion-translocation across the membrane, thus allowing chemiosmotic energy generation. Protonpumping Rnf clusters have been integrated into the 
energy-conserving systems of Clostridium tetani [58], Clostridium kluyveri [38] and Acetobacterium woodii [59]. In C. sticklandii, reduced ferredoxin is formed in several metabolic processes such as the highly exergonic NADH-dependent reduction of crotonyl-CoA to butyryl-CoA in the butyrate and lysine fermentation pathways $[60,61]$ and the reactions catalysed by numerous ferredoxin oxidoreductases present in the C. sticklandii genome (Figure 1A). In addition to the possible ion-gradient driven chemiosmotic ATP generation via the Rnf complex, C. sticklandii possesses a $\mathrm{Na}^{+}$-dependent F0F1-ATPase and an additional V-ATPase. This is a quite unusual combination for bacteria [62]. Further energy conservation can be provided by a reaction catalyzed by a putative methylmalonyl-CoA decarboxylase. This membrane-bound protein couples the decarboxylation of methylmalonyl-CoA to the efflux of $\mathrm{Na}^{+}$ions, which re-enter the cell by the ATPase systems. A third chemiosmotic energy-conserving process is catalyzed by a membrane-bound pyrophosphatase [63]. An alternative energy-conserving system in anaerobes is provided by the presence of hydrogenases. The C. sticklandii genome contains two cytoplasmic iron-only hydrogenases (HydA and HymABC). The multisubunit hydrogenase $\mathrm{Hym} A B C$ exhibits similarity with the hydrogenase identified in Eubacterium acidaminophilum [64]. The structural gene for the second hydrogenase HydA is clustered together with genes coding for a formate dehydrogenase (FdhA, FdhB), making it possible for these proteins to act as a formate hydrogen lyase. Formate addition to the culture medium increased growth efficiency of C. sticklandii [12]. A formate transporter is present, as in E. acidaminophilum [64]. Electrons from the oxidation of formate to $\mathrm{CO}_{2}$ are transferred directly to the hydrogenase via ferredoxin to reduce $\mathrm{H}^{+}$ions to hydrogen inside the cell, thus lowering the proton concentration. Genes encoding the hydrogenase maturation proteins HydE, HydF, and HydG are localized downstream from the hydrogenase gene hydA.

\section{The glycine synthase/glycine reductase pathway and the Wood-Ljungdahl pathway: a surprising combination}

A particular metabolic feature of C. sticklandii is the simultaneous presence of genes encoding the complete Wood-Ljungdahl pathway and the glycine synthase (reversed gcv system)/glycine reductase pathway (Figure 1A). The Wood-Ljungdahl pathway is the main characteristic feature of acetogenic bacteria, making them capable of using $\mathrm{CO}_{2}$ as an electron acceptor. Many of these organisms can also grow autotrophically utilizing $\mathrm{CO}_{2}$. In certain purine- or amino acid-fermenting "acetogenic" bacteria acetate is synthesized from C1-carbon units via the glycine synthase/glycine reductase pathway $[65,66]$. It has been reported that the glycine synthase/glycine reductase pathway is not present in acetogenic bacteria containing the Wood-Ljungdahl pathway [67]. Since C. sticklandii possesses both pathways, a possible function of the Wood-Ljungdahl pathway may be the formation of methylene-THF from $\mathrm{CO}_{2}$, which is then used in the glycine synthase/glycine reductase pathway as described for amino acid-fermenting "acetogenic" bacteria. However, this does not lead to a net energy conservation, since ATP formed via the glycine reductase reaction is consumed for the activation of formate to form formyl-THF. It also seems likely that the whole Wood-Ljungdahl pathway is turned off completely under fermentative conditions [68], since metabolites involved in fermentation can act as terminal electron acceptors, thus removing the electrons necessary for the reduction of $\mathrm{CO}_{2}$ to acetate. Therefore, we assume that $C$. sticklandii cannot grow autotrophically like acetogenic bacteria.

Two main processes of chemiosmotic energy conservation have been described in acetogenic bacteria. The first, observed in Moorella thermoacetica, assumes that the methylene-THF reductase is associated with the cytoplasmic membrane and is coupled to a proton translocating NADH:quinone oxidoreductase with cytochromes and menaquinone. The second energy conservation system, described in $A$. woodii, suggests that the methyl group transfer from methyl-THF to the carbon monoxide dehydrogenase/acetyl-CoA synthase by a membrane-bound corrinoid protein is accompanied by an efflux of $\mathrm{Na}^{+}$ions. In both systems energy is conserved by the ion-motive force-driven ATPase system. In $C$. sticklandii the methylene-THF reductase does not seem to be attached to the cytoplasmic membrane, cytochromes are missing, menaquinone is not synthesized and membrane-bound corrinoids are absent. Recently, Schmidt et al. proposed that in $A$. woodii energy could be conserved in the Wood-Ljungdahl pathway by the membrane-bound Rnf reaction [59]. As mentioned above, the Rnf complex catalyzes an electron flow from reduced ferredoxin to $\mathrm{NAD}^{+}$, a reaction coupled to proton or sodium efflux. Reduced $\mathrm{NAD}^{+}$often provides electrons for the reductive steps of the methyl-branch of the Wood-Ljungdahl pathway. The regeneration of NADH via the reduced ferredoxin-dependent Rnf complex leads to chemiosmotic energy conservation. Appropriate experiments are necessary to determine whether either the Wood-Ljungdahl pathway or the glycine synthase/glycine reductase pathway, or both, are active in C. sticklandii.

In any case, it was interesting to see whether other microorganisms contain this particular combination. For this purpose, the characteristic protein sequences of the carbon monoxide dehydrogenase/acetyl-CoA synthase from the Wood-Ljungdahl pathway, the gcv- system and 
the glycine reductase from C. sticklandii were compared with completely sequenced bacterial genomes and those available as a draft. This analysis reveals that, in addition to $C$. sticklandii, only four bacterial species contain the genes coding for these proteins (Additional file 8): Alkaliphilus metalliredigens, Carboxydothermus hydrogenoformans, Clostridium carboxidivorans, and several strains of Clostridium difficile. Of these C. hydrogenoformans can grow autotrophically using carbon monoxide as the sole carbon and energy source and presumably synthesizes acetate from $\mathrm{CO}_{2}$ via the Wood-Ljungdahl pathway $[69,70]$. Altogether, these results clearly demonstrate that the coexistence of genes encoding the WoodLjungdahl pathway and the glycine synthase/glycine reductase pathway, as found in C. sticklandii, is not widespread.

\section{Conclusions}

Sequencing and annotation of the small genome of C. sticklandii confirms that this organism derives most of its carbon and energy from the fermentation of amino acids. In particular, the genes coding for D-proline and glycine reductases, characteristic enzymes of the Stickland reaction, are both present in this genome. The inspection of the D-proline reductase cluster leads to the discovery that PrdC is a selenoprotein and contributes to a detailed understanding of the mechanism of D-proline reductase. Analysis of amino acid utilization demonstrates that C. sticklandii degrades amino acids in a preferential way. The data generated shows that threonine, arginine and serine are the best sources of carbon and energy, whereas aromatic amino acids, glutamate, aspartate and alanine are not or hardly utilized. The degradation of lysine, a process which has been described as a major energy source, is only performed in the stationary growth phase. The genome-scale metabolic reconstruction of $C$. sticklandii provides valuable information about energy conservation in anaerobic microorganisms. Beside SLP, the characteristic energy-conserving method in fermentative bacteria, C. sticklandii possesses several systems to conserve energy by ETP. These include a membrane-associated ion pumping Rnf complex as well as the rather unusual combination of a F- and V-type ATPase. Another particular feature is the simultaneous presence of two $\mathrm{CO}_{2}$-fixation pathways: the WoodLjungdahl and the glycine synthase/glycine reductase pathways. Comparative genomics reveals that the amino acid degradation pathways of $C$. sticklandii present homologies with the environmental bacteria, A. metalliredigens and $A$. oremlandii OhILAs, and with the pathogenic clostridial strains, C. botulinum and especially $C$. difficile. Since $C$. diffcile shares a similar energy metabolism with $C$. sticklandii, these two organisms could serve as an excellent model for comparative studies of the lifestyle of pathogenic and non-pathogenic organisms.

\section{Methods}

\section{Genome sequencing and assembly}

A Sanger/pyrosequencing hybrid approach was used for whole-genome sequencing of C. sticklandii DSM 519. A shotgun library was constructed with a $10 \mathrm{~kb}$ size fraction obtained by mechanical shearing of total genomic DNA. The fragments were cloned into vector pCNS (pSU18 derived). Insert ends of the recombinant plasmids (21504 reads, $\sim 6 \times$ coverage) were sequenced by dye terminator chemistry using ABI3730 sequencers. Next, total genomic sheared DNA was sequenced $(\sim 21 \times$ coverage) using the Roche GS20 sequencer. For the assembly, we used the Arachne "HybridAssemble" (Broad Institute, http://www.broad.mit.edu) that combines the 454 contigs with Sanger reads.

\section{Gene prediction and annotation}

Annotation was performed using MaGe [44,71], which allows graphic visualization of the C. sticklandii DSM 519 annotations enhanced by a simultaneous representation of synteny groups in other genomes chosen for comparisons. Coding sequences (CDS) likely to encode proteins were predicted with AMIGene [72].

\section{Growth conditions and amino acid analysis}

C. sticklandii strain DSM 519 was grown at $37^{\circ} \mathrm{C}$ under a nitrogen atmosphere in the synthetic medium described by Wagner and Andreesen which contains required trace elements such as selenite and tungstate [33]. Cultures for the analysis of amino acid utilization were prepared in a medium containing all amino acids (except alanine and aspartate) at a concentration of 2 $\mathrm{mM}$, as well as the cofactor and trace element solutions used in the synthetic medium [33]. Samples were collected at different growth phases (exponential, the end of the exponential, stationary, and the end of the stationary phases), centrifuged for $10 \mathrm{~min}$ at $20,000 \mathrm{~g}$, and the supernatant was analyzed by LC-MS. The majority of the amino acids were analyzed directly and some were derivatized with o-phthalic aldehyde and $\mathrm{N}$-acetyl L-cysteine as described by Brückner et al. [73]. Additional file 5 shows the amino acid utilization profiles obtained.

\section{Mass spectrometry}

Chromatographic separations of free amino acids were performed using an Aquity UPLC BEH C18 column $(150 \times 2.1 \mathrm{~mm}, 1.7 \mu \mathrm{m}$; Waters $)$. The amino acids were eluted with $10 \mathrm{mM}$ ammonium acetate solution containing $0.1 \%$ formic acid (A) and methanol containing $0.1 \%$ 
formic acid (B). Chromatographic separations of the derived amino acids were performed employing an XBridge C18 column $(150 \times 2.1 \mathrm{~mm}, 5 \mu \mathrm{m}$; Waters $)$. These were eluted with a $30 \mathrm{mM}$ ammonium acetate solution (A) and methanol (B). All LC-MS analyses were performed using a LTQ/Orbitrap high resolution mass spectrometer coupled to an Accella LC system (Thermo-Fisher Scientific).

\section{Nucleotide sequence accession number}

The genome sequence reported in this article has been deposited in the EMBL database [EMBL:FP565809]. The genome annotation and features are available at http:// www.genoscope.cns.fr/agc/microscope/Anaeroscope.

\section{List of abbreviations}

ATP: adenosine triphosphate; CoA or CoASH: coenzyme A; ETP: electron-transport phosphorylation; fd: ferredoxin; FMN: flavin mononucleotide; gcv-system: glycine cleavage system; LC-MS: Liquid Chromatography-Mass Spectrometry; MaGe: Magnifying Genomes; NADH: reduced nicotinamide adenine dinucleotide; NADPH: reduced nicotinamide adenine dinucleotide phosphate; Ppase: pyrophosphatase; Rnf: protein involved in Rhodobacter capsulatus nitrogen fixation; Sec: selenocysteine; SLP: substrate-level phosphorylation; THF: tetrahydrofolate; WGS: whole genome shotgun.

\section{Additional material}

Additional file 1: Genomic and metabolic features of C. sticklandii compared with other clostridia. All data are extracted from the MaGe annotations except those of Clostridium sporogenes, which are from the NCBI database. C.stick: Clostridium sticklandii DSM 519; C.acet: Clostridium acetobutylicum ATCC 824; C.beij: Clostridium beijerinckii NCIMB 8052; C.botu: Clostridium botulinum A Hall; C.diff: Clostridium difficile 630; C.kluv: Clostridium kluyveri DSM 555; C.novy: Clostridium novyi NT; C.perf: Clostridium perfringens ATCC 13124; C.phyt: Clostridium phytofermentans ISDg; C.teta: Clostridium tetani E88; C.ther: C. thermocellum ATCC 27405; C.spor: C. sporogenes ATCC 1557; A.meta: Alkaliphilus metalliredigens QYMF; A.orem: Alkaliphilus oremlandii OhILAs; M.ther: Moorella thermoacetica ATCC 39073. *2-ketoacid ferredoxin oxidoreductases.

Additional file 2: $C$. sticklandii synteny conservation and Bidirectional Best Hit percentages with the most closely related clostrial species. Synteny conservation is given by the percentage of CDS in synteny between C. sticklandii and the complete sequenced genomes.

Additional file 3: Characteristic gene products of $C$. sticklandii, their protein symbols and their corresponding labels. This is a listing of all genes/proteins that are discussed in the text.

Additional file 4: Comparative analysis of amino acid degradation pathways in several clostridial species. Blast searches (criteria: $30 \%$ identity over at least $80 \%$ of the length of the reference protein) were performed to determine the presence of the key enzymes in the microorganisms. All protein sequences were taken from C. sticklandii with two exceptions: the sequence of methylaspartate mutase was from Clostridium cochlearium, and that of 2-hydroxyglutaryl-COA dehydratase from Acidaminococcus fermentans. C.stick: Clostridium sticklandii DSM 519; C.acet: Clostridium acetobutylicum ATCC 824; C.beij: Clostridium beijerinckii NCIMB 8052; C.botu: Clostridium botulinum A Hall; C.diff: Clostridium difficile 630; C.Kluv: Clostridium kluyveri DSM 555; C.novy: Clostridium novyi NT; C.perf: Clostridium perfringens ATCC 13124; C.phyt: Clostridium phytofermentans ISDg; C.teta: Clostridium tetani E88; C.ther: C. thermocellum ATCC 27405; C.spor: C. sporogenes ATCC 15579; A.meta: Alkaliphilus metalliredigens QYMF; A.orem: Alkaliphilus oremlandii OhILAs; M.ther: Moorella thermoacetica ATCC 39073; C.coch: Clostridium cochlearium; A.ferm: Acidaminococcus fermentans DSM 20731. The genome of Clostridium cochlearium is not yet sequenced.

Additional file 5: LC-MS analyses of amino acid utilization during growth. For each amino acid, its presence in the medium was checked at different growth phases (colour graphs). The growth kinetic is represented by a yellow graph. Citrulline, ornithine, alanine and aspartate (indicated by a black frame) were not added to the medium. They appeared as intermediates or products of metabolism from other amino acids. Since the utilization of methionine was not discussed in the article, the graph for this amino acid is not shown.

Additional file 6: Cell growth of C. sticklandii in three types of media with different amino acid composition. Graph 1: medium containing amino acids that are catabolized in the exponential phase (Pro, Asn, Thr, Ser, Arg, Cys); Graph 2: medium containing amino acids that are catabolized in the exponential and stationary phase (Pro, Asn, Thr, Ser, Arg, Cys, Leu, Iso, Met, Gln, His, Lys); Graph 3: the amino acid combination of this medium is equivalent to the second medium, complemented with amino acids apparently not metabolized (Pro, Asn, Thr, Ser, Arg, Cys, Leu, Iso, Met, Gln, His, Lys, Trp, Val, Phe, Glu). Tyrosine was added to each medium.

Additional file 7: Comparison of the D-proline reductase gene cluster from different clostridial species. White arrows indicate hypothetical proteins or those presumed not to be involved in the D-proline reductase reaction. The letter " $U$ " indicates the presence of selenocysteine. In Clostridium botulinum A Hall, a part of the cluster is duplicated.

Additional file 8: Presence of characteristic enzymes of the glycine synthase (Gcv proteins)/glycine reductase (Grd proteins) and WoodLjungdahl (CODH proteins) pathway in microbial genomes. Complete and WGS microbial genomes (as of May 6th, 2010) were scanned for some selected characteristic orthologous genes coding for carbon monoxide dehydrogenase/acetyl-CoA synthetase (WoodLjungdahl pathway), glycine reductase (Stickland reaction), and the glycine synthase (gcv-system) using a blastx search with stringent criteria ( $40 \%$ of positive residues over at least $80 \%$ of the length of the reference protein). For each target genome, the presence/absence of each protein was summarized in a $1 / 0$ vector. Those WGS microbial genomes which do not contain all genes of both pathways are omitted.

\section{Acknowledgements}

This work was supported by the Commissariat à l'Energie Atomique (CEA). The authors thank Claudine Médigue, David Vallenet, Antoine Danchin, Susan Cure and Alexander Smith for improving the manuscript as well as the Genoscope sequencing team for the sequence data.

\section{Author details}

${ }^{1}$ CEA, DSV, Institut de Génomique, Genoscope, 2 rue Gaston Crémieux, F91057 Evry, France. ${ }^{2}$ CNRS-UMR 8030 F-91057 Evry, France. ${ }^{3}$ UEVE, Université d'Evry, F-91057 Evry, France. ${ }^{4}$ Institut Pasteur, 28 rue du Dr. Roux, F-75724 Paris cedex 15, France. ${ }^{5}$ Institute of Biology/Microbiology, University of Halle, Kurt-Mothes-Str. 3, D-06120 Halle, Germany.

\section{Authors' contributions}

AK, NF and GNC performed genome and comparative analysis and coordinated experimental procedures. SC carried out the growth experiments. ST analysed amino acid utilization by LC-MS. NP contributed to the experimental procedures. AL contributed to elaboration of the clostridial database Anaeroscope. MG constructed shotgun library for sequencing. VB carried out the assembly and finishing of the genome. EP performed bioinformatic analyses. ST, AL and VB participated in writing sections of the manuscript. MS, DL, and JW critically reviewed the manuscript. GNC and JRA 
contributed to writing of the manuscript. AK and NF wrote the manuscript All authors read and approved the final manuscript.

Received: 17 May 2010 Accepted: 11 October 2010

Published: 11 October 2010

\section{References}

1. Elsden SR, Hilton MG: Amino acid utilization patterns in clostridial taxonomy. Arch Microbiol 1979, 123:137-41.

2. Mead GC: The amino acid-fermenting clostridia. J Gen Microbiol 1971, 67:47-56.

3. Collins MD, Lawson PA, Willems A, Cordoba JJ, Fernandez-Garayzabal J, Garcia P, Cai J, Hippe H, Farrow JA: The phylogeny of the genus Clostridium: proposal of five new genera and eleven new species combinations. Int J Syst Bacteriol 1994, 44:812-26.

4. Kim J, Hetzel M, Boiangiu CD, Buckel W: Dehydration of (R)-2-hydroxyacylCoA to enoyl-CoA in the fermentation of alpha-amino acids by anaerobic bacteria. FEMS Microbiology Reviews 2004, 28:455-68.

5. Kim J, Darley DJ, Buckel W, Pierik AJ: An allylic ketyl radical intermediate in clostridial amino-acid fermentation. Nature 2008, 452:239-42.

6. Stadtman TC: Discoveries of vitamin B12 and selenium enzymes. Annu Rev Biochem 2002, 71:1-16.

7. Stickland LH: Studies in the metabolism of the strict anaerobes (genus Clostridium): The chemical reactions by which $\mathrm{Cl}$. sporogenes obtains its energy. Biochem J 1934, 28:1746-59.

8. Andreesen JR: Glycine reductase mechanism. Curr Opin Chem Biol 2004, 8:454-61.

9. Arkowitz RA, Abeles RH: Mechanism of action of clostridial glycine reductase: isolation and characterization of a covalent acetyl enzyme intermediate. Biochemistry 1991, 30:4090-7.

10. JenneyJr FE, Verhagen MF, Cui X, Adams MW: Anaerobic microbes: oxygen detoxification without superoxide dismutase. Science 1999, 286:306-9.

11. Nisman B: The Stickland reaction. Bacteriol Rev 1954, 18:16-42.

12. Stadtman TC, WhiteJr FH: Tracer studies on ornithine, lysine, and formate metabolism in an amino acid fermenting Clostridium. J Bacteriol 1954, 67:651-7.

13. Stadtman TC, Barker HA: Studies on the methane fermentation. X. A new formate-decomposing bacterium, Methanococcus vannielii. J Bacteriol 1951, 62:269-80.

14. Andreesen J, Bahl H, Gottschalk G: Introduction to the Physiology and Biochemistry of the Genus Clostridium. In Clostridia. Edited by: Minton N, Clarke D. New York and London: Plenum Press; 1989:27-62.

15. Barker HA: Amino acid degradation by anaerobic bacteria. Annu Rev Biochem 1981, 50:23-40.

16. Stadtman TC: On the metabolism of an amino acid fermenting Clostridium. J Bacteriol 1954, 67:314-20.

17. Elsden SR, Hilton MG: Volatile acid production from threonine, valine, leucine and isoleucine by clostridia. Arch Microbiol 1978, 117:165-72.

18. Elsden SR, Hilton MG, Waller JM: The end products of the metabolism of aromatic amino acids by Clostridia. Arch Microbiol 1976, 107:283-8

19. Schäfer R, Schwartz AC: Catabolism of purines in clostridium sticklandii. Zentralbl Bakteriol Orig A 1976, 235:165-72.

20. Schwartz AC, Schäfer R: New amino acids, and heterocyclic compounds participating in the Stickland reaction of Clostridium sticklandii. Arch Microbiol 1973, 93:267-76

21. Stadtman TC, McClung LS: Clostridium sticklandii nov. spec. J Bacteriol 1957, 73:218-9.

22. Jackson S, Calos M, Myers A, Self WT: Analysis of proline reduction in the nosocomial pathogen Clostridium difficile. J Bacteriol 2006, 188:8487-95.

23. Golovchenko N, Belokopytov B, Chuvil'skaya N, Akimenko V: Minimal synthetic culture medium for the bacterium Clostridium sticklandii. Applied Biochemistry and Microbiology 1985, 20:430-434

24. Zein F, Zhang Y, Kang YN, Burns K, Begley TP, Ealick SE: Structural insights into the mechanism of the PLP synthase holoenzyme from Thermotoga maritima. Biochemistry 2006, 45:14609-20.

25. Golovchenko NP, Belokopytov BF, Akimenko VK: [Glucose metabolism in Clostridium sporogenes and Clostridium sticklandii bacteria]. Mikrobiologija 1983, 52:869-74.

26. Fisher E, Dawson AM, Polshyna G, Lisak J, Crable B, Perera E, Ranganathan M, Thangavelu M, Basu P, Stolz JF: Transformation of inorganic and organic arsenic by Alkaliphilus oremlandii sp. nov. strain OhILAs. Ann N Y Acad Sci 2008, 1125:230-41.

27. Ye Q, Roh Y, Carroll SL, Blair B, Zhou J, Zhang CL, Fields MW: Alkaline anaerobic respiration: isolation and characterization of a novel alkaliphilic and metal-reducing bacterium. Appl Environ Microbiol 2004, 70:5595-602

28. Woolfolk CA, Woolfolk BS, Whiteley HR: 2-oxypurine dehydrogenase from Micrococcus aerogenes. I. Isolation, specificity, and some chemical and physical properties. J Biol Chem 1970, 245:3167-78.

29. Fonknechten N, Perret A, Perchat N, Tricot S, Lechaplais C, Vallenet D, Vergne C, Zaparucha A, Le Paslier D, Weissenbach J, et al: A conserved gene cluster rules anaerobic oxidative degradation of L-ornithine. J Bacteriol 2009, 191:3162-7.

30. Kenklies J, Ziehn R, Fritsche K, Pich A, Andreesen JR: Proline biosynthesis from L-ornithine in Clostridium sticklandii: purification of delta1pyrroline-5-carboxylate reductase, and sequence and expression of the encoding gene, proC. Microbiology 1999, 145:819-26.

31. Andreesen JR: Glycine metabolism in anaerobes. Antonie Van Leeuwenhoek 1994, 66:223-37.

32. Zindel U, Freudenberg W, Rieth $M$, Andreesen JR, Schnell J, Widdel F: Eubacterium acidaminophilum sp.nov., a versatile amino aaciddegrading anaerobe producing or utilizing $\mathrm{H} 2$ or formate. Arch Microbiol $1988,150: 254-266$

33. Wagner M, Andreesen JR: Purification and characterization of threonine dehydrogenase from Clostridium sticklandii. Arch Microbiol 1995, 163:286-90.

34. Costilow RN, Laycock L: Ornithine cyclase (deaminating). Purification of a protein that converts ornithine to proline and definition of the optimal assay conditions. J Biol Chem 1971, 246:6655-60.

35. Chang CH, Frey PA: Cloning, sequencing, heterologous expression, purification, and characterization of adenosylcobalamin-dependent Dlysine 5, 6-aminomutase from Clostridium sticklandii. J Biol Chem 2000, 275:106-14.

36. Kreimeyer A, Perret A, Lechaplais C, Vallenet D, Medigue C, Salanoubat M, Weissenbach J: Identification of the last unknown genes in the fermentation pathway of lysine. J Biol Chem 2007, 282:7191-7.

37. Stadtman T: Lysine metabolism by Clostridia. Advances in Enzymology and Related Areas of Molecular Biology 1973, 38:413-448.

38. Seedorf $H$, Fricke WF, Veith B, Brüggemann $H$, Liesegang $H$, Strittmatter $A$, Miethke M, Buckel W, Hinderberger J, Li F, et al: The genome of Clostridium kluyveri, a strict anaerobe with unique metabolic features. Proc Natl Acad Sci USA 2008, 105:2128-33.

39. Buckel W, Barker HA: Two pathways of glutamate fermentation by anaerobic bacteria. J Bacteriol 1974, 117:1248-60.

40. Buckel W: Unusual enzymes involved in five pathways of glutamate fermentation. Appl Microbiol Biotechnol 2001, 57:263-73.

41. Fryer TF, Mead GC: Development of a selective medium for the isolation of Clostridium sporogenes and related organisms. J Appl Bacteriol 1979, 47:425-31.

42. Cone JE, Del Rio RM, Davis JN, Stadtman TC: Chemical characterization of the selenoprotein component of clostridial glycine reductase: identification of selenocysteine as the organoselenium moiety. Proc Natl Acad Sci USA 1976, 73:2659-63.

43. Turner DC, Stadtman TC: Purification of protein components of the clostridial glycine reductase system and characterization of protein $A$ as a selenoprotein. Arch Biochem Biophys 1973, 154:366-81.

44. Vallenet D, Engelen S, Mornico D, Cruveiller S, Fleury L, Lajus A, Rouy Z, Roche D, Salvignol G, Scarpelli C, et al: MicroScope: a platform for microbial genome annotation and comparative genomics. Database (Oxford) 2009, 2009, bap021.

45. Kabisch U: Proteinchemische und molekularbiologische Charakterisierung der D-Prolin-Reduktase aus Clostridium sticklandii. PhD thesis Halle: Martin-Luther University 2001

46. Kabisch U, Gräntzdörffer A, Schierhorn A, Rücknagel KP, Andreesen JR, Pich A: Identification of D-proline reductase from Clostridium sticklandii as a selenoenzyme and indications for a catalytically active pyruvoyl group derived from a cysteine residue by cleavage of a proprotein. $J$ Biol Chem 1999, 274:8445-54. 
47. Eversmann B: Molekulare und biochemische Untersuchungen zu Komponenten der D-Prolin-Reduktase und Glycin-Reduktase von Clostridium sticklandii. PhD thesis Halle: Martin-Luther University 2004

48. Lee BC, Dikiy A, Kim HY, Gladyshev VN: Functions and evolution of selenoprotein methionine sulfoxide reductases. Biochim Biophys Acta 2009, 1790:1471-7

49. Seto B, Stadtman TC: Purification and properties of proline reductase from Clostridium sticklandii. J Biol Chem 1976, 251:2435-9.

50. Lovitt RW, Kell DB, Morris JG: Proline reduction by Clostridium sporogenes is coupled to vectorial proton ejection. FEMS Microbiology Letters 1986 36:269-273.

51. Seto B: The Stickland reaction. In Diversity in Bacterial Respiratory Systems. Edited by: Knowles C. Boca Raton: CRC Press; 1980:2:50-64.

52. Uhde A: Wachstumsphysiologische Untersuchungen zum Abbau von Aminosäuren und mögliche Funktion eines elektronentransferierenden Flavoproteons bei Clostridium sticklandii. Dipl thesis Göttingen: GeorgAugust University 1990.

53. Schwartz AC, Müller W: NADH-dependent reduction of D-proline in Clostridium sticklandii. Reconstitution from three fractions containing $\mathrm{NADH}$ dehydrogenase, D-proline reductase, and a third protein factor. Arch Microbiol 1979, 123:203-8.

54. Bednarski $B$, Andreesen JR, Pich A: In vitro processing of the proproteins GrdE of protein B of glycine reductase and PrdA of D-proline reductase from Clostridium sticklandii: formation of a pyruvoyl group from a cysteine residue. Eur J Biochem 2001, 268:3538-44.

55. Gröbe T, Reuter M, Gursinsky T, Söhling B, Andreesen JR: Peroxidase activity of selenoprotein GrdB of glycine reductase and stabilisation of its integrity by components of proprotein GrdE from Eubacterium acidaminophilum. Arch Microbiol 2007, 187:29-43.

56. Imlay JA: Iron-sulphur clusters and the problem with oxygen. $\mathrm{Mol}$ Microbiol 2006, 59:1073-82.

57. Hillmann F, Fischer RJ, Saint-Prix F, Girbal L, Bahl H: PerR acts as a switch for oxygen tolerance in the strict anaerobe Clostridium acetobutylicum. Mol Microbiol 2008, 68:848-60.

58. Brüggemann $H$, Baumer S, Fricke WF, Wiezer A, Liesegang $H$, Decker I, Herzberg C, Martinez-Arias R, Merkl R, Henne A, et al: The genome sequence of Clostridium tetani, the causative agent of tetanus disease. Proc Natl Acad Sci USA 2003, 100:1316-21.

59. Schmidt $\mathrm{S}$, Biegel $\mathrm{E}$, Müller $\mathrm{V}$ : The ins and outs of $\mathrm{Na}(+)$ bioenergetics in Acetobacterium woodii. Biochim Biophys Acta 2009, 1787:691-6.

60. Herrmann G, Jayamani E, Mai G, Buckel W: Energy conservation via electron-transferring flavoprotein in anaerobic bacteria. J Bacterio/ 2008 190:784-91.

61. Li F, Hinderberger J, Seedorf H, Zhang J, Buckel W, Thauer RK: Coupled ferredoxin and crotonyl coenzyme $\mathrm{A}(\mathrm{Co} A)$ reduction with $\mathrm{NADH}$ catalyzed by the butyryl-CoA dehydrogenase/Etf complex from Clostridium kluyveri. J Bacteriol 2008, 190:843-50.

62. Müller V, Grüber G: ATP synthases: structure, function and evolution of unique energy converters. Cell Mol Life Sci 2003, 60:474-94.

63. Serrano A, Perez-Castineira JR, Baltscheffsky M, Baltscheffsky H: H+-PPases: yesterday, today and tomorrow. IUBMB Life 2007, 59:76-83

64. Graentzdoerffer A, Rauh D, Pich A, Andreesen JR: Molecular and biochemical characterization of two tungsten- and selenium-containing formate dehydrogenases from Eubacterium acidaminophilum that are associated with components of an iron-only hydrogenase. Arch Microbiol 2003, 179:116-30.

65. Dürre $P$, Andreesen J: Pathway of carbon dioxide reductionto acetate without a net energy requirement in Clostridium purinolyticum. FEMS Microbiol Lett 1982, 15:51-56.

66. Schneeberger A, Frings J, Schink B: Net synthesis of acetate from CO2 by Eubacterium acidaminophilum through the glycine reductase pathway. FEMS Microbiology Letters 1999, 177:1-6.

67. Fuchs G: $\mathrm{CO} 2$ fixation in acetogenic bacteria: variations on a theme. FEMS Microbiology Reviews 1986, 39:181-213.

68. Drake $H$, Küsel K: How the diverse physiologic potential of acetogens determine their in situ realities. In Biochemistry and Physiology of Anaerobic Bacteria. Edited by: Ljungdahl L, Adams M, Barton L, Ferry J, Johnson M. New York: Springer; 2003:171-190.

69. Svetlitchnyi V, Dobbek H, Meyer-Klaucke W, Meins T, Thiele B, Romer P, Huber R, Meyer O: A functional Ni-Ni-[4Fe-4S] cluster in the monomeric
acetyl-CoA synthase from Carboxydothermus hydrogenoformans. Proc Natl Acad Sci USA 2004, 101:446-51.

70. Wu M, Ren Q, Durkin AS, Daugherty SC, Brinkac LM, Dodson RJ, Madupu R, Sullivan SA, Kolonay JF, Haft DH, et al: Life in hot carbon monoxide: the complete genome sequence of Carboxydothermus hydrogenoformans Z-2901. PLoS Genet 2005, 1:e65.

71. Vallenet D, Labarre L, Rouy Z, Barbe V, Bocs S, Cruveiller S, Lajus A, Pascal G, Scarpelli C, Medigue C: MaGe: a microbial genome annotation system supported by synteny results. Nucleic Acids Res 2006, 34:53-65.

72. Bocs S, Cruveiller S, Vallenet D, Nuel G, Medigue C: AMIGene: Annotation of Mlcrobial Genes. Nucleic Acids Res 2003, 31:3723-6.

73. Brückner H, Wittner R, Godel H: Automated enantioseparation of amino acids by derivatization with o-phthaldialdehyde and n-acylated cysteines. J Chromatogr 1989, 476:73-82.

\section{doi:10.1186/1471-2164-11-555}

Cite this article as: Fonknechten et al:: Clostridium sticklandii, a specialist in amino acid degradation:revisiting its metabolism through its genome sequence. BMC Genomics 2010 11:555.

\section{Submit your next manuscript to BioMed Central and take full advantage of:}

- Convenient online submission

- Thorough peer review

- No space constraints or color figure charges

- Immediate publication on acceptance

- Inclusion in PubMed, CAS, Scopus and Google Scholar

- Research which is freely available for redistribution 